\title{
VirtuaLabQ - Ambiente para a Prática Experimental de Transformações Químicas
}

\author{
Saulo Ramos ${ }^{1}$, Edson P. Pimentel ${ }^{1}$ \\ ${ }^{1}$ Universidade Federal do ABC (UFABC) - Santo André - SP - Brasil \\ Centro de Matemática, Computação e Cognição - CMCC \\ Grupo de Pesquisa INTERA \\ \{srdcp@hotmail.com, edson.p.pimentel@gmail.com\}
}

\begin{abstract}
The availability of laboratories for conducting practical activities, required in Chemistry Teaching, is a difficulty faced at different levels of education. This article aims to present the VirtuaLabQ, an environment with gamification elements to support the experimental practice of Chemical Transformations. The article also presents the results of a case study that reports the use of the environment with students in the subject of Chemical Transformations University $X Y X$, in order to evaluate the environment's potential in supporting teaching and learning.
\end{abstract}

Resumo. A disponibilidade de laboratórios para a realização de atividades práticas, necessárias no Ensino de Química, é uma dificuldade encontrada nos diferentes níveis de ensino. Este artigo tem por objetivo apresentar o VirtuaLabQ, um ambiente com elementos de gamificação para apoiar a prática experimental de Transformações Químicas. $O$ artigo também apresenta um estudo de caso que relata o uso do ambiente com alunos na disciplina de Transformações Químicas da Universidade Federal do ABC, com o objetivo de avaliar as potencialidades do ambiente no apoio ao ensinoaprendizagem.

\section{Introdução}

Uma das dificuldades encontradas no Ensino de Química é a disponibilização de laboratórios para a realização de atividades práticas. São necessários horários específicos para que o aluno realize estes experimentos, com supervisão de técnicos, disponibilidade de recursos como reagentes, equipamentos e outros.

Giordan (1999) afirma que é do conhecimento dos professores de Química o fato de que a experimentação desperta um forte interesse entre os alunos e que aulas realizadas nos laboratórios didáticos aumentam a capacidade de aprendizado dos alunos, pois estas facilitam o entendimento do assunto abordado. Almeida et al. (2008) afirmam que estes experimentos facilitam a compreensão dos conceitos de natureza da ciência, auxiliam no desenvolvimento de atitudes científicas e no diagnóstico de concepções não-científicas além de despertar o interesse pela ciência. A pesquisa realizada em Almeida et al. (2008) mostra que os alunos se sentem mais motivados para aprender Química, quando se faz o uso de aulas práticas e dinâmicas. 
Silva (2011) expõe cinco problemas enfrentados no ensino de Química: (i) a formação do professor; (ii) a metodologia; (iii) o salário dos educadores; (iv) o desinteresse dos alunos; (v) a diminuição na formação dos licenciados. Silva (2011) ainda afirma que algumas escolas do ensino médio não possuem laboratórios adequados para as aulas experimentais e, quando possuem, carecem de professores específicos para a prática experimental, o que torna insuficiente o aprendizado quando utilizando somente aulas teóricas. A disponibilidade de laboratórios para a prática experimental de química no ensino superior também pode ser considerada insuficiente, ainda mais para que o aluno possa realizar exercícios de forma mais autônoma.

Este trabalho pressupõe que a ampliação de recursos para aumentar a prática experimental de química pode ser suprida pela disponibilização de ferramentas como laboratórios virtuais que tornem o aprendizado acessível utilizando-se de recursos que motivem o aluno a realizar as práticas experimentais.

Este artigo tem por objetivo apresentar o VirtuaLabQ, um ambiente para apoiar a prática experimental de Transformações Químicas. A fim de também atacar o problema do "desinteresse dos alunos" citado por Silva (2011) o ambiente implementa elementos de gamificação, tais como: pontuação, classificação e uso de avatar. O artigo apresenta também os resultados do uso do ambiente com alunos na disciplina de Transformações Químicas da Universidade Federal do ABC, com o objetivo de avaliar as potencialidades do ambiente no apoio ao ensino-aprendizagem.

O artigo está organizado como segue: a seção 2 apresenta a fundamentação teórica abordando a Prática de transformações Químicas além de conceitos e elementos de gamificação; a seção 3 descreve o ambiente VirtuaLabQ e suas funcionalidades; a seção 4 apresenta a avaliação da ferramenta em um estudo de caso; Por fim, a seção 5 apresenta as considerações finais e delineia os trabalhos futuros.

\section{Fundamentação Teórica}

Esta seção apresenta, de forma breve, a fundamentação teórica central para o desenvolvimento do ambiente: o conteúdo de Transformações Químicas abordado e os conceitos e elementos de gamificação. Apresenta ainda alguns trabalhos correlatos..

\subsection{Prática de Transformações Químicas}

Para o desenvolvimento do laboratório virtual elegeu-se os conteúdos da disciplina "Transformações Químicas", obrigatória para todos os 1500 estudantes que ingressam no curso Bacharelado em Ciência e Tecnologia da Universidade Federal do ABC (UFABC). Os dois principais motivos para a escolha foram: (i) a abordagem prática da disciplina e (ii) a possibilidade de experimentar o ambiente na própria disciplina.

A disciplina de Transformações Químicas tem como objetivos de acordo com a sua ementa (Ribeiro, 2015): (a) Fornecer os fundamentos básicos de química para a compreensão de fenômenos envolvendo a relação entre as transformações que ocorrem no meio ambiente e as propriedades dos materiais envolvidos; (b) Correlacionar as propriedades macroscópicas dos materiais com propriedades microscópicas, permitindo ao estudante tomar consciência do papel desempenhado pela Química nas Ciências e também de sua importância na sociedade e no cotidiano e (c) Introduzir as técnicas básicas de laboratório. 
A bibliografia básica da disciplina é composta pelos seguintes autores: Atkins e Jones (2011), Kotz, Treichel e Weaver (2010) e Brady, Russell , e Holum (2012). A disciplina é desenvolvida em doze semanas e as atividades práticas envolvem a realização de quatro experimentos listados no quadro 1 e descritos em Ribeiro (2015).

Quadro 1 - Atividades Práticas em Transformações Químicas

\begin{tabular}{|l|l|}
\hline \multicolumn{1}{|c|}{ Experimentos da Aula Prática } & \multicolumn{1}{|c|}{ Tópicos Envolvidos } \\
\hline Semelhante dissolve semelhante? & $\begin{array}{l}\text { Ligações Químicas, Interações Moleculares, Reações } \\
\text { Químicas. }\end{array}$ \\
\hline Produzindo um air-bag & Reações Químicas. Estequiometria. \\
\hline Aquecendo a água em um camping & $\begin{array}{l}\text { Calor envolvido em transformações químicas (Entalpia). } \\
\text { Transferência de calor. }\end{array}$ \\
\hline Velocidade das reações & Cinética Química. Leis da velocidade de reações \\
\hline
\end{tabular}

\subsection{Elementos de Gamificação}

Para Deterding et al. (2011), gamificação é: "o uso de elementos de jogos em contextos não associados a jogos". Há uma distinção entre jogo e diversão, Caillois (1996) define esta diferença como Paidia e Ludus: Paidia significa a brincadeira espontânea, improvisada, como crianças que criam regras na hora da brincadeira. Ludus significa o jogo controlado na forma de regras, manuais, limites e instruções.

Groh (2012) diz que no contexto da gamificação é abordado o design de elementos de jogos, ou seja, Ludus. Embora a gamificação possua similaridades com os jogos como pontos, níveis e troféus, os jogos necessitam de elementos como a fantasia, a dramatização e vencedores que não são requisitos da gamificação.

Um bom exemplo de aplicativo gamificado é o Foursquare (disponível em https://pt.foursquare.com/) que a partir de geolocalização em dispositivos móveis, que recomenda lugares como bares e restaurantes. Para cada local que o usuário $\mathrm{X}$ chega, pontos são acumulados e se $\mathrm{X}$ for o usuário que mais acessa determinado lugar, torna-se o prefeito daquele local.

Simões et al. (2013) propõem uma visão do sistema gamificado, com bases nos elementos apresentados por Zichermann (2012), constituído pelo conjunto de componentes fundamentais e os elementos de jogos, conforme o Quadro 2.

\section{Quadro 2 - Componentes e Elementos de Jogos (Fluxo e Diversão) Fonte: Adaptado de Simões et al. (2013)}

\begin{tabular}{|c|c|}
\hline $\begin{array}{c}\text { Componentes } \\
\text { Fundamentais }\end{array}$ & Elementos de Jogos \\
\hline $\begin{array}{c}\text { Comunicação e Recompensas } \\
\text { (Feedback \& Rewards) }\end{array}$ & Pontos, Tabelas de Liderança, Barras de Progresso, Insignias, Troféus \\
\hline Envolvente Social (Friends) & Partilhar Troféus, Convidar Amigos, dar/solicitar/trocar bens virtuais \\
\hline $\begin{array}{c}\text { Experiência de Jogo } \\
\text { (Gameplay) }\end{array}$ & $\begin{array}{c}\text { Níveis, Objetivos Intermediários, Objetivos Claros, Repetição após } \\
\text { falha (fun failure), Regras, Economia Virtual, Escalonamenta de } \\
\text { Recompensas }\end{array}$ \\
\hline
\end{tabular}

Vale ressaltar que existem diversas aplicações da gamificação no mundo real. Por exemplo, professores que presenteiam os seus alunos com "estrelinhas" a cada atividade concluída ou a cada aula em que o aluno está presente. Isto motiva a criança a participar e frequentar mais a escola. Hoje, os estudantes têm uma relação muito próxima com a tecnologia e convivem diariamente com elementos de gamificação. 
Nesse sentido, pode tornar-se pouco motivador realizar atividades escolares apenas com "lápis e papel”, competindo com as diversas alternativas do mundo da tecnologia.

\subsection{Trabalhos Correlatos}

Em relação ao ensino de química alguns jogos ou ambientes gamificados podem ser encontrados na literatura. O Quadro 3 apresenta alguns deles:

Quadro 3 - Trabalhos Correlatos: jogos relacionados ao ensino de química

\begin{tabular}{|c|l|}
\hline Autor & \multicolumn{1}{|c|}{ Descrição do Trabalho } \\
\hline Lima et al. (2011) & $\begin{array}{l}\text { Jogo baseado em um jogo de cartas comercialmente chamado de } \\
\text { Super Trunfo. O tema central são as cartas que possuem elementos } \\
\text { químicos da tabela periódica. }\end{array}$ \\
\hline Silva e Rebouças (2011) & $\begin{array}{l}\text { Jogo desenvolvido para auxiliar no ensino-aprendizagem de Química } \\
\text { com foco nas nomenclaturas químicas. O trabalho visa o uso dos } \\
\text { dispositivos móveis, principalmente os tablets. }\end{array}$ \\
\hline Bertolini et al. (2013) & $\begin{array}{l}\text { A ferramenta "iLaboratory" atua como um simulador de laboratório } \\
\text { para a reprodução de alguns experimentos de química, de forma } \\
\text { interativa, através de dispositivos móveis. }\end{array}$ \\
\hline Nunes et al. (2014) & $\begin{array}{l}\text { Laboratório Virtual de Química baseado no Mundo Virtual OpenSim. } \\
\text { O usuário interage com o laboratório em um mundo virtual 3D e } \\
\text { realiza os experimentos através de uma interface com o Moodle onde } \\
\text { há um NPC (Non Player Character) que guia o usuário durante a } \\
\text { execução dos experimentos. }\end{array}$ \\
\hline
\end{tabular}

\section{O Ambiente VirtuaLabQuim}

Esta seção apresenta o ambiente VirtuaLabQ e os aspectos relacionados ao seu desenvolvimento como a arquitetura do ambiente, metodologia de aplicação de gamificação e interface.

\subsection{Arquitetura do Ambiente}

A Figura 1 apresenta de forma esquemática a arquitetura do ambiente VirtuaLabQ composto pelos módulos de Entrada, Menu Principal e Experimentos.

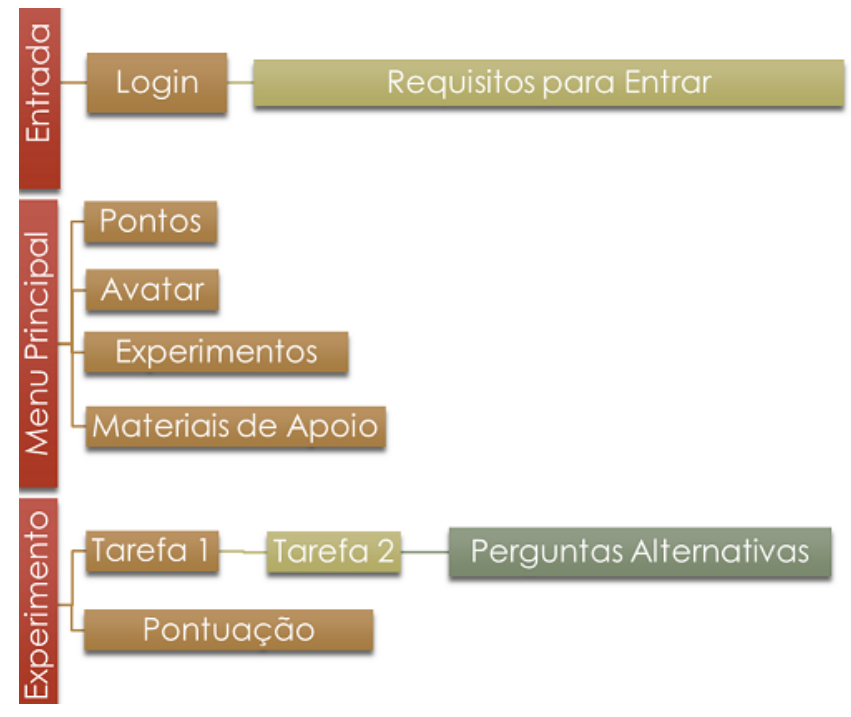

Figura 1 - Organização do VirtuaLabQ 
No menu principal o usuário pode visualizar seus pontos, seu avatar e selecionar os experimentos a realizar, bem como acessar o material de apoio.

\subsection{Modelo de Gamificação}

Este trabalho utilizou como base o modelo de referência de Simões et al. (2013) para a aplicação da gamificação. O quadro 4 apresenta a aplicação do modelo no que diz respeito aos seguintes itens: (1) Caracterização do Contexto não Lúdico; (2) Identicação do Objetivos e (3) Seleção de Elementos de Jogos.

Quadro 4 - Aplicação do Modelo de Simões et al (2013) no VirtuaLabQ

\begin{tabular}{|c|c|}
\hline $\begin{array}{l}\text { 1.1 Identificar a natureza do } \\
\text { contexto (real ou virtual) }\end{array}$ & $\begin{array}{l}\text { Real. Experimentos Realizados em } \\
\text { Laboratório de Química }\end{array}$ \\
\hline 1.2 Identificar as atividades & $\begin{array}{l}\text { Realização dos Experimentos: } \\
\text { Mistura de Reagentes, Soluções, Montagem } \\
\text { de Bancada para a Realização dos Experimentos } \\
\text { Manuseio de Equipamentos e Vidrarias }\end{array}$ \\
\hline $\begin{array}{l}\text { 1.3 Definir os comportamentos } \\
\text { alvo }\end{array}$ & $\begin{array}{l}\text { O Laboratório deve motivar os alunos } \\
\text { a executarem os experimentos e } \\
\text { instigá-los a realizar todos os experimentos }\end{array}$ \\
\hline $\begin{array}{l}\text { 1.4 Caracterizar o perfil dos } \\
\text { jogadores }\end{array}$ & $\begin{array}{l}\text { Alunos de Transformações Químicas da } \\
\text { Universidade Federal do ABC. } \\
\text { Média de idade } 19 \text { anos. }\end{array}$ \\
\hline \multicolumn{2}{|l|}{ 2. Identificar os Objetivos } \\
\hline $\begin{array}{l}\text { 2.1 Definir os Objetivos em função } \\
\text { dos comportamentos-alvo }\end{array}$ & $\begin{array}{l}\text { Desenvolvimento de um Laboratório Virtual } \\
\text { que contenha elementos de jogos que } \\
\text { motivem o aluno a participar e executar os } \\
\text { experimentos }\end{array}$ \\
\hline 2.2 Quantificar os Objetivos & $\begin{array}{l}\text { 1. Pesquisar elementos que influenciem na } \\
\text { motivação e que possam fazer parte da proposta } \\
\text { do Laboratório Virtual } \\
\text { 2. Incorporá-los à ideia do Laboratório } \\
\text { 3. Validá-los através de um Estudo de Caso }\end{array}$ \\
\hline \multicolumn{2}{|l|}{ 3. Selecionar os Elementos de Jogos } \\
\hline 3.1 Comunicação e Recompensas & Correção das Atividades / Pontuação \\
\hline 3.2 Envolvente Social & Ranking / Avatar \\
\hline 3.3 Experiência de Jogo & $\begin{array}{l}\text { Interatividade com Elementos / Divisão por } \\
\text { Experimentos / Animações / Efeitos de Tela / } \\
\text { Imagens }\end{array}$ \\
\hline
\end{tabular}

\subsection{Interface do Ambiente}

Após efetuar o login no ambiente utilizando os dados da rede social Facebook o ambiente exibe a tela de requisitos para ingresso no laboratório. Após verificar e marcar todos os itens necessários para adentrar no Laboratório Virtual como se estivesse entrando em um laboratório real o usuário terá acesso às atividades disponíveis.

Na tela de Menu Principal, apresentada na Figura 2, são exibidos os pontos do usuário, avatar ou foto, os experimentos que podem ser realizados e também links para os materiais de apoio necessários para que o usuário realize os experimentos. 


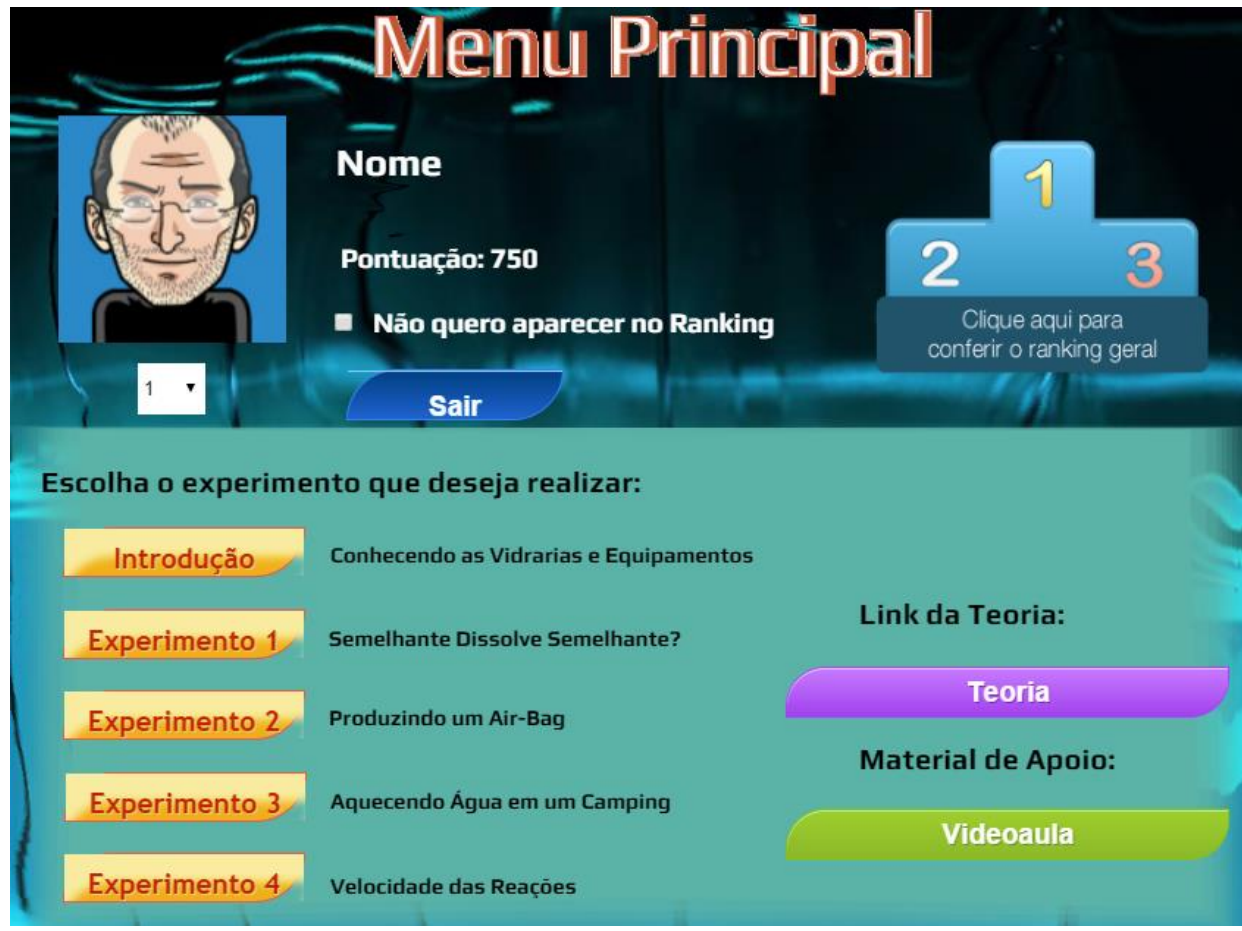

Figura 2 - Menu Principal do VirtuaLabQ

Ao selecionar um experimento o usuário é direcionado para a tela específica na qual são apresentadas missões ou tarefas para que o experimento seja executado. A Figura 3 apresenta a tela do experimento 1 - Semelhante dissolve Semelhante?

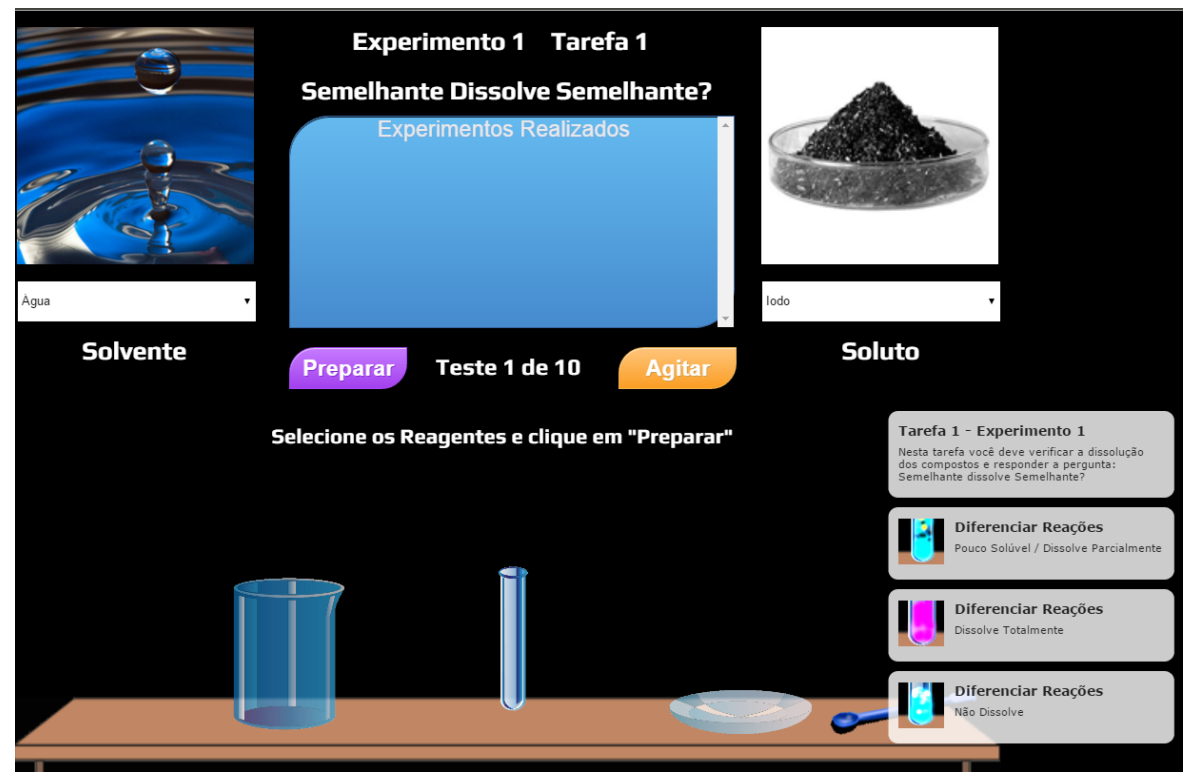

Figura 3 - Tela do Experimento 1 - Semelhante dissolve semelhante?

Após o usuário terminar a realização do experimento, são exibidas perguntas alternativas para avaliar os conhecimentos do usuário. A Figura 4 apresenta um recorte da tela de questões relativa ao experimento 2 - Produzindo um Air Bag. 


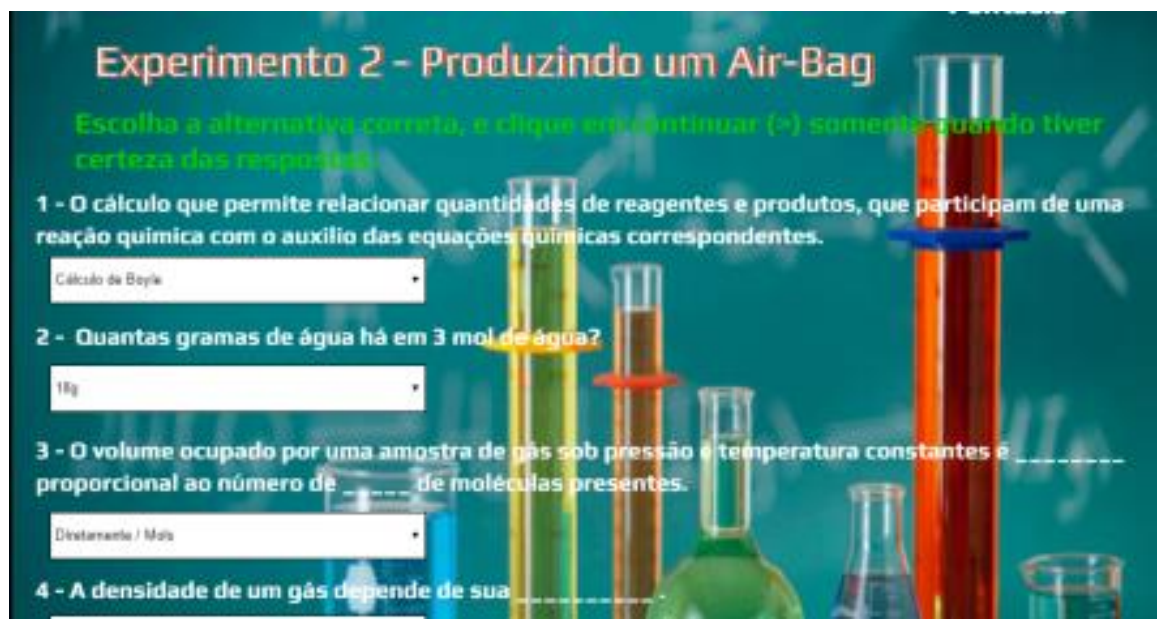

Figura 4 - Questões para Avaliação do Experimento 2.

Após responder às questões de avaliação do experimento o ambiente exibe a pontuação que o usuário obteve no experimento selecionado, um botão para verificar o ranking $e$ outro botão para avaliar a atividade realizada no Laboratório Virtual, conforme figura 5.

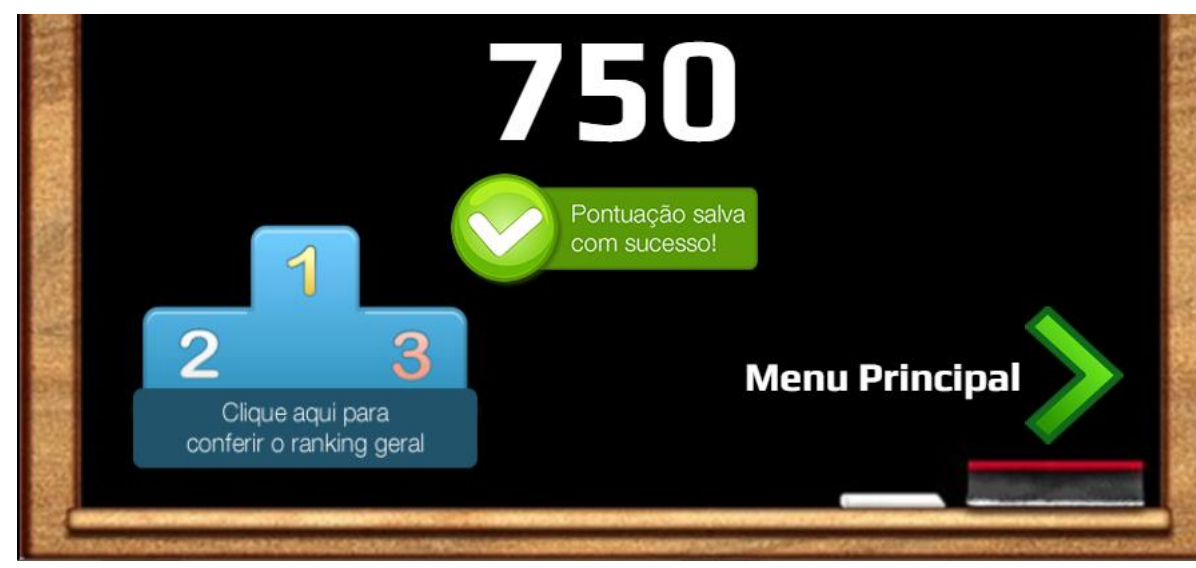

Figura 5 - Recorte da Tela de Pontuação do Experimento

\subsection{Tecnologias Utilizadas}

As seguintes ferramentas foram utilizadas no desenvolvimento do VirtuaLabQ:

- Scirra Construct 2 (C2) - ferramenta para aplicações 2D utilizada para o desenvolvimento dos experimentos, animações, efeitos e etc.

- Brackets - software para a criação e edição de códigos para a Web (HTML, CSS, JavaScript e PHP) utilizado para desenvolver a comunicação com o Banco de Dados, a página de Ranking e a Área do Professor.

\section{Estudo de Caso - Avaliando as Potencialidades do VirtuaLabQ}

A fim de avaliar a usabilidade do ambiente VirtuaLabQ e também o seu potencial em cumprir os objetivos quando comparado aos experimentos realizados num laboratório real, esboçou-se um estudo de caso para aplicação com alunos da disciplina de Transformações Químicas do curso de Bacharelado em Ciência e Tecnologia da 
Universidade Federal do ABC. O projeto de aplicação foi aprovado pelo Comitê de Ética em Pesquisa da universidade.

\subsection{Universo da Investigação}

Para a realização do estudo de caso foram convidados alunos de duas turmas do período diurno e duas turmas do período noturno. Cada turma possuía 30 alunos matriculados, somando-se 120 alunos. Todos os alunos cursavam a disciplina de Transformações Químicas da UFABC e os conteúdos ministrados bem como as atividades experimentais eram os mesmos.

Os alunos foram instruídos a utilizar o Laboratório Virtual antes da realização dos experimentos em um Laboratório Real, e preencher questionários adequados para cada experimento realizado nos diferentes laboratórios (Real e Virtual).

\subsection{Instrumentos de Pesquisa}

Para o estudo de caso foram utilizados dois questionários avaliativos: (i) O primeiro questionário deveria ser preenchido logo após a realização do experimento no VirtuaLabQ respondendo perguntas quanto às suas contribuições para o aprendizado, a disposição dos botões e objetos, a facilidade de uso da ferramenta, etc. (ii) O segundo questionário deveria ser preenchido logo após a realização do experimento em um Laboratório Real, respondendo a perguntas quanto ao grau de contribuição da execução do experimento primeiramente no laboratório virtual, a similaridade e dificuldade da manipulação dos reagentes nos laboratórios real e virtual, a necessidade de realizar o experimento novamente no laboratório real uma vez que executado no laboratório virtual, entre outros.

\section{3 . Resultados e Análise}

Dos 120 alunos convidados 32 participaram de todas as etapas, respondendo a todos os questionários e foram considerados nessa análise. Quanto ao perfil dos alunos, $75 \%$ eram homens e $25 \%$ eram mulheres, destes $93,75 \%$ nunca haviam cursado a disciplina e $6,25 \%$ responderam que já haviam cursado a disciplina ao menos uma vez.

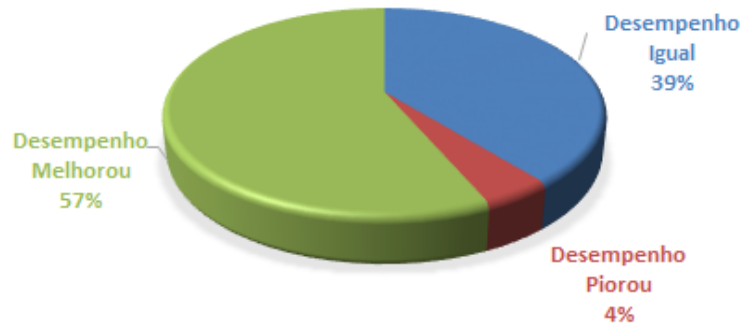

(a)

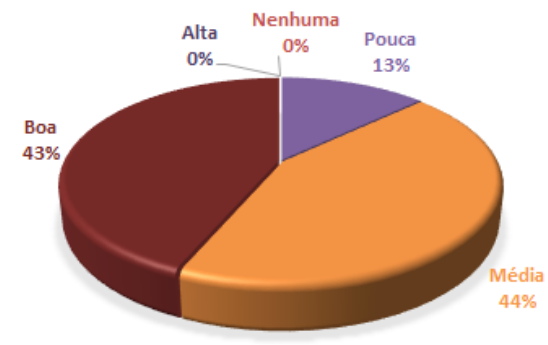

(b)

Figura 6 - (a) Comparação de desempenho VirtuaLabQ x Real

(b) Contribuição do uso do VirtuaLabQ

Ao perguntar aos alunos sobre "seu desempenho no laboratório real em relação ao seu desempenho no laboratório virtual" 57\% indicaram que o desempenho melhorou. $\mathrm{Na}$ mesma direção, 87\% $(44+43=87)$ dos alunos afirmaram que o VirtuaLabQ 
contribuiu positivamente para uma posterior realização do experimento em um laboratório real. Os dois gráficos estão disponíveis na Figura 6(a) e 6(b).

A figura 7 apresenta um resumo da avaliação do VirtuaLabQ em relação às dicas, usabilidade, semelhança com o laboratório real, a contribuição da realização dos experimentos virtualmente e a avaliação quanto ao desempenho na realização do experimento presencialmente.

\section{Dicas}

Usabilidade

Semelhança

Contribuição

Desempenho
$81 \%$ dos alunos afirmaram que as dicas poderiam ser de boa utilidade durante a realização do experimento porém $94 \%$ não as utilizaram

$69 \%$ dos alunos responderam positivamente, demonstrando que as ferramentas utilizadas para a produção dos efeitos, equipamentos e resultados foram selecionadas corretamente para o desenvolvimento deste projeto

$79 \%$ semelhante em relação aos equipamentos e $83 \%$ em relação aos Reagentes

$75 \%$ dos alunos afirmaram que o Laboratório Virtual contribuiu significativamente para o aprendizado nesta disciplina

$57 \%$ afirmou que o desempenho melhorou

$87 \%$ afirmou que o Laboratório Virtual aumentou a facilidade em realizar os experimentos no Laboratório Real

Figura 7 - Resumo da Avaliação do VirtuaLabQ

\section{Considerações Finais}

O VirtuaLabQ foi desenvolvido numa perspectiva de que a ampliação de recursos para aumentar a prática experimental de química pode ser suprida pela disponibilização de ferramentas como laboratórios virtuais cujo uso independem da presença de técnicos de laboratórios, de reagentes, etc. A inclusão de elementos de gamificação no ambiente teve por objetivo aumentar a motivação para seu uso. Nesse sentido, no estudo de caso realizado como primeira avaliação do ambiente, os elementos que aparentemente mais contribuíram para a motivação dos alunos foram a Pontuação e o Ranking. Os dados coletados a partir do Log (arquivo que registra as ações do usuário) demonstraram que alguns alunos executaram os experimentos diversas vezes, possivelmente para buscar maiores pontuações. Outra possibilidade é que tenham refeito as atividades para treinar, aprender, e melhor se preparar para as atividades no laboratório presencial.

Apesar de ter contribuído para o aprendizado, os alunos (67\%) afirmaram que há a necessidade de realizar novamente os experimentos no Laboratório Real. Algumas motivações são a limitação do Laboratório Virtual em manusear os equipamentos, como também a impossibilidade de mensurar alguns fatores que influenciam na realização do experimento como pureza dos reagentes, temperatura ambiente, transferência de energia (calor) nos equipamentos alterando alguns resultados enquanto a prática experimental deve considerar estes fatores.

Para trabalhos futuros, diversas funcionalidades podem ser implementadas no VirtuaLabQ tais como: utilização de mundos virtuais 3D e de Realidade Aumentada, considerar fatores que influenciem nos experimentos, citados também pelos alunos como as perdas de energia, pureza dos reagentes e introdução de outros elementos de jogos. Assim outros estudos podem ser feitos, como teste de hipótese e a utilização de metodologias para avaliação de objetos de aprendizagem. 
Agradecimentos: A CAPES por concessão de bolsa de estudos durante a pesquisa.

\section{Referências Bibliográficas}

Almeida, E. C. S., da Silva, M. D. F. C., de Lima, J. P., da Silva, M. L., Braga, C. D. F., \& Brasilino, M. D. G. A. (2008). Contextualização do ensino de química: motivando alunos de ensino médio." X Encontro de Extensão. UFPB - PRAC

Atkins, Peter e Jones, Loretta (2011) Princípios de Química, Questionando a vida e o meio ambiente, Bookman, Porto Alegre, $5^{\text {a }} \mathrm{E}$.

Bertolini, C. T., Braga, J. C., Pimentel, E., \& Ramos, S. (2013). Laboratório Virtual Interativo para reprodução de Experimentos de Química através de Dispositivos Móveis. In Anais do Simpósio Brasileiro de Informática na Educação (Vol. 24, No. 1).

Brady, James E.; Russell , Joel W. e Holum , John R. (2012) Química - a Matéria e Suas Transformações, $5^{\text {a }}$ ed, Volume 1 e 2, LTC Editora, Rio de Janeiro.

Caillois, R. (1961) "Man, play, and games. [S.1.]: University of Illinois Press."

Deterding, S., Khaled, R., Nacke, L. E., \& Dixon, D. (2011, May). Gamification: Toward a definition. In CHI 2011 Gamification Workshop Proceedings (pp. 12-15).

Groh, F. (2012) Gamification: State of the art definition and utilization. Institute of Media Informatics Ulm University, v. 39.

Kotz, John; Treichel , Paul e Weaver, Gabriela (2010) Química Geral e Reações Químicas, Vol. 1 e 2, Cengage Learning, São Paulo.

Lima, E. C., Mariano, D. G., Pavan, F. M., Lima, A. A., \& Arçari, D. P. (2011). Uso de Jogos Lúdicos Como Auxilio Para o Ensino de Química.Revista Eletrônica Educação em Foco.

Nunes, A. S.; Adorni, D. S. (2010) "O ensino de química nas escolas da rede pública de ensino fundamental e médio do município de Itapetinga-Ba: O olhar dos alunos." Encontro Dialógico Transdisciplinar - Enditrans - Educação e conhecimento científico, Vitória da Conquista, BA.

Oliveira, Celina Couto, José Wilson da Costa, and Marcia Moreira. (2001) "Ambientes informatizados de aprendizagem: produção e avaliação de software educativo." Papirus,

Ribeiro, Anderson O. (2015) Transformações Químicas. Disponível em https://sites.google.com/site/transformacoesquimicasufabc/. Acesso em 22 de Julho de 2015.

Santos, A. O., Silva, R. P., Andrade, D., \& Lima, J. P. M. (2013). Dificuldades e motivações de aprendizagem em Química de alunos do ensino médio investigadas em ações do (PIBID/UFS/Química). Scientia Plena, 9(7 (b)).

Silva, Airton Marques. (2011) "Proposta para tornar o ensino de química mais atraente." Revista de Química Industrial. RQI - $2^{\circ}$ trimestre

Silva, Diego Lopes Marques, and Ayla Débora Dantas S. Rebouças. (2011) "MyQuímica-Um jogo para auxiliar no ensino-aprendizagem das nomenclaturas químicas." Anais do XXII SBIE - XVII WIE

Simões J., Rebeca Redondo, Ana Vilas, Ademar Aguiar. Proposta de modelo de referência para aplicação de gamification em ambientes de aprendizagem social. Challenges 2013 Proceedings of the VIII International Conference on ICT in Education

Zichermann, Gabe, and Christopher Cunningham.(2011) "Gamification by design: Implementing game mechanics in web and mobile apps. " O'Reilly Media, Inc., 2011. 